\title{
Designing of Single Band Four Antenna Array for 5G Mobile Applications using MISO Technique
}

\author{
https://doi.org/10.3991/ijim.v15i20.23747 \\ Padmapriya T. ${ }^{(凶)}$, Manikanthan S.V. \\ Melange Academic Research Associates, Puducherry, India \\ padmapriyaa85@pec. edu
}

\begin{abstract}
A single-band four-antenna array is proposed in this paper for Multiple Input Single Output (MISO) mechanisms in 5G applications. Different L-shaped slot antennas based on step-impedance resonators were also used in the existing MISO antenna array Stepped Impedance Resonator (SIRs). The proper single-resonance for each antenna part could be calculated by measuring the SIR's impedance ratio. Good impedance matching could be achieved by fine-tuning the antenna's micro strip antenna feature's orientation. The findings show that over the long-term evolution (LTE) bands 52 (3358-3640 MHz) and 20, each antenna system with a simulated cost efficiency of more than 61 percent had a calculated return loss of more than $10 \mathrm{~dB}$ and a measured inter-element isolation of more than $10.2 \mathrm{~dB}(5250-5985 \mathrm{MHz})$. Moreover, the calculated Envelope Correlation Coefficient (ECC) for any two antenna parts is greater than 0.2, and the suggested MISO antenna array achieves a virtual channel capacity of 42.5 $\mathrm{bps} / \mathrm{Hz}$ 's in both transmission band. In the presence of Specific Anthropomorphic Mannequins (SAM) in the head and human brain, in particular, the MISO antenna array can ensure adequate radiation and MISO accuracy.
\end{abstract}

Keywords-multiple input single output (MISO), stepped impedance resonator (SIR), long term evolution (LTE), return loss, specific anthropomorphic mannequin (SAM) head, inter element isolation, impedance, single band, channel capacity, step - impedance resonator

\section{$1 \quad$ Introduction}

$5 \mathrm{G}$ is the fifth generation of wireless communications network infrastructure standards in telecommunications, which wireless carriers recently implemented progressively in 2019, and is the intended complement to the $4 \mathrm{G}$ networks that connect directly to most present cell phones [1]. According to the GSM organization, 5G projects are likely to have more than 1.7 thousand followers by 2025 [2]. 5G networks are network protocols, like our predecessors, in the frequency band is segregated into cells called regional remote communities. Both $5 \mathrm{G}$ wireless technology in a cell connect to another internet and telecommunications network through a local antenna in the grid. 
5G networks are wireless sensor network, like those predecessors, in which the area from coverage is fragmented into cells called small regional centres.

Because of the faster download speeds [1] new networks had greater capability, gradually reaching 10 gigabits per second (Gbit/s). Because of all the hyper threading, networks are expected to not only accommodate disposal, such as existing cellular networks, but also to be used by laptops and desktop computers as general internet service providers, interacting with other ISPs including innovations in the Internet of Things (IoT) and device aspects by the use of the wired internet. The new networks that really include wireless devices equipped support $5 \mathrm{G}$ are just unable to use $4 \mathrm{G}$ smart phones. Radio waves with higher amplitude than previous cellular polysaccharides are commonly used to achieve intensified performance. Higher-frequency radio waves, however, have a shorter physical range that encourages smaller local cells to be unable to be useful. On up to three frequency bands, $5 \mathrm{G}$ networks interface to for narrow service: Massive, medium, and low. A 5G network should include networks of up to three various tissues, each unique to the facilities configurations of each organization, each setting up a separate trade off in forms of download velocity vs distance and designations. At their venue or via the highest speed antenna within their range, reconnect to the network including $5 \mathrm{G}$ cellphones and capacitive touchscreens.

600-850 MHz, a similar frequency spectrum used in $4 \mathrm{G}$ cellular telephones, can be used for low-band $5 \mathrm{G}$, with download rates significantly higher than 4G: There is a diameter and coverage region for the antennas in a low-band network, which is 30-250 megabits per second (Mbit/s) [3] equivalent to 4G towers. Mid-band 5G employs microwaves operating at 2.5-3.7 GHz to provide speeds of 100-900 Mbit/s, with each cellular tower delivering data over a distance of many miles. This is the most regularly determined service level and is supposed to be available in all metropolitan areas by 2020. There is still no low-band implementation for continents, making this the minimum degree of network. At the very bottom of the transceiver band, high-band $5 \mathrm{G}$ uses 25-39 GHz frequencies, though higher frequencies can also be used in the future. It also incorporates Gigabit per second (Gbit/s) download capacities in the range, analogous to the cable internet. There is, however, a more narrowed transmitter bandwidth (mm wave or $\mathrm{mm} \mathrm{W}$ ), allowing for very many small cells [4]. These varieties of instruments, such as windows and walls, have problems going through them. These cells are projected to be integrated only in dense urban environments and places where women gather, such as sports stadiums, and conference centres, because their higher cost. The speeds discussed above the real findings acquired in 2020 were those that set to increase speeds during roll-out [3].

$5 \mathrm{G}$ networks are digital radio networks which distribute the spectrum of companies available in small cell-called geographical fields. Audiovisual analogue signals are embedded into the telephone, converted and transmitted as a bit stream through the use of an analog-to-digital converter. A pool of frequencies regenerated in other radio wave cells, a corresponding antenna array, and a low-power automated transceiver in the cell specified by the transceiver are used by $5 \mathrm{G}$ wireless equipment in a cell to communicate. A high-bandwidth optical transmission or wireless backhaul link connects the local antennas to the phone network and the internet. A telecommunications system that transitions from one cell to another is then comfortably 'started to turn over' to the new 
cell, as in other cell networks. Up to a million devices per city block can be sponsored by $5 \mathrm{G}$, because only up to 100,000 devices per square kilo $[5,6]$ are sponsored by $4 \mathrm{G}$. $4 \mathrm{G} \mathrm{LTE}$ capability would also be appropriate for the newest $5 \mathrm{G}$ mobile networks, as $4 \mathrm{G}$ is now used by new networks to initially connect to the device, as well as in situations where $5 \mathrm{G}$ communication is not convenient [7]. For enhanced performance, as well as higher throughput, most network operators use frequency bands. Since there are fewer transmitters than microwaves, the cells must be smaller [8]. Closing borders are more complicated when millimetre conditions are present [9].

One of the hottest debates in recent years towards the rise of the digital age for communications has been the 5 th generation $(5 \mathrm{G})$ of mobile communications. For any wireless devices and systems, antennas, filters, amplifiers, mixers, and so on are essential aspects for the RF front-end. The prices of necessary variants of high-performance and modified antennas and circuits is thus growing drastically. Most approaches, however, mainly have difficulty attracting the restrictive bandwidth, radiation pattern, capacity, and cost parameters of $5 \mathrm{G}$ mobile communications. For researchers from both academia and industry, therefore, high-performance antennas and circuits for $5 \mathrm{G}$ android phones are very interesting. This special issue requests high-quality contributions to the design of microwave and millimeter-wave antennas and circuits for developing communication systems. The below Figure 1 shows the $5 \mathrm{G}$ network communication.

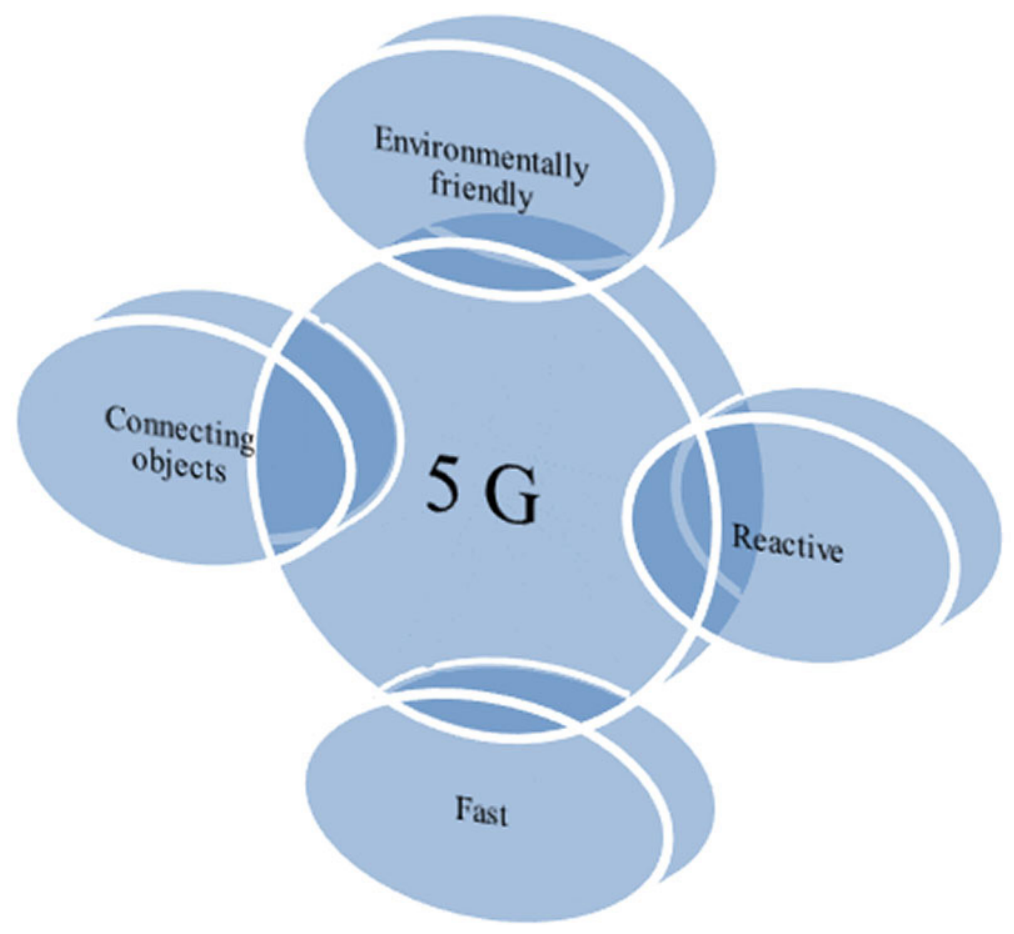

Fig. 1. Schematic diagram of $5 \mathrm{G}$ network 
Because of all the enormous the prosperity of modern communication networks, individual people have proposed an incredibly high rate of transmission of information. As a result, it is critical for modern wireless communication technologies to have a much higher channel capacity, which allows good the adoption of $5 \mathrm{G}$ communications. In the near future, the world will usher in Communications including 5G. As one of the crucial 5G networking technologies, multiple MIMO (Multiple Input and Output) systems that rely on multiple antennas are well known must enhance the capacity of the channel and strengthen communication reliability. Compared to $4 \mathrm{G}$ communications, $5 \mathrm{G}$ communications might achieve a higher communication range, shorter time delay, and greater receiver sensitivity. As a result, the $2 * 2$ and $4 * 4$ MISO antenna arrays have initially focused on meeting the specific rules. Therefore, several experiments was carried out on the $4 * 4$ MISO Designs of communication systems that are equipped to be used for mobile $5 \mathrm{G}$ terminals [23], [24].

The 52 long-term evolution (LTE) band (3358-3640 MHz) has been approved among the sub- $6 \mathrm{GHz}$ bands [10]. Therefore, numerous LTE band 42 MISO antenna arrays have been optimized. Thus, other LTE band 52 MISO antenna arrays have been developed [11]. However, the demand for the incoming $5 \mathrm{G}$ multiband communications cannot be thoroughly supplied by the MISO antenna array residing merely on LTE band 42. Although somewhat unlicensed, the undoubtedly and competitive storage of LTE band $46(5150-5925 \mathrm{MHz})$ is for 5G MIMO applications that could be used [12], [22]. Promising candidates for the improvement of the MISO the multimode resonator, loaded resonator without multi-stub and resonator of level impedance are an antenna array in mobile terminals (SIR). Coordinating a large number of forms of energy because of all the limited space in a contemporary setting is impossible. Also ensuring good isolation and radiation control, commonly-sized smartphones.

Various techniques for remembering the multiband MISO In the literature, an 5G mobile terminal antenna array was also studied. Many multi-mode resonators or three micro compositions are the cornerstone of these designs. A 4-antenna to serve on LTE band 52 and LTE band 46 is required and also MISO array has been analysed. Two independent radiators are responsible for different bands, namely the slot antenna and the control antenna. The developers got a return loss of more than $6 \mathrm{~dB}$ and a one to 0.15 envelope correlation coefficient. A high channel capability has an effect as well. Vertically directed antenna features, on the other hand, are more efficient in terms of thickness and length, manufactured products. An increasing number of MISO triple band antennas Specific radiators are used in an inverted-shaped antenna, a longer L-shaped slot antenna, and a shorter L-shaped slot antenna. By a different network of interconnected radiators, each band is obtained. A deficit of return of much more than $6 \mathrm{~dB}$ had also been achieved and an ECC of less than 0.15 had also been achieved.

\section{Related works}

Abdullah, M., et al., [13] explained systematic development of an eight-element high-performance antenna array for a $2.6 / 3.5 \mathrm{GHz}$ band $5 \mathrm{G}$ mobile terminal. Depending on the embedded monopole patch antennas module in the metal or ground framework, 
the planned series of eight apparatus lecturers correlates at $2.6 \mathrm{GHz}$ in normal operation. The antenna array is installed from four antennas combined just next to the edges of the integrated circuit towards the early part of the long edge ground plane with provided pairs of vertically mounted slot antennas. This combination of antenna elements produced a variety of configurations and permitted the smartphone to interpret the signal in another direction. There are several other approved bands based on the $-10 \mathrm{~dB}$ return loss of the indispensable $2.4 \mathrm{GHz}$ to $3.66 \mathrm{MHz}$ infrared region. This apart from power balance and appropriate diversity capabilities, simple MIMO performance metrics like the envelope correlation coefficient or ECC were just 0.2 for any two large link speeds achieving the standard average of less than 0.5 , and the mean effective gain or MEG ratio of any two signals met the minimum average of less than $3 \mathrm{~dB}$. Since current mobile requirements necessitate thin handsets, it was feasible to use the above lightweight multiple antenna configuration for future smart phones because the conductive sheet or frame is used and the vertically mounted middle antenna would not require additional chassis or ground space. The effect of a person's hand or a human's hand on a multiple antenna array is often investigated in order to determine a consumer's cell device use. The nominal MIMO transmission mode is $34.25 \mathrm{bps} / \mathrm{hz}$ and three of the central $2 * 2$ MIMO operations are anticipated for business growth.

Ren, Z., \& Zhao, A. [14] described that Fifth-generation (5G) mobile terminal application. It is recommended that only single, self-decoupled antenna pairs be based on the antenna system. High absorption dual-band antenna pairs could be accomplished by establishing a single propagation unit for the two successive antenna units. In both the $3.5 \mathrm{GHz}(3.4-3.6 \mathrm{GHz})$ and $4.9 \mathrm{GHz}(4.8-5.0 \mathrm{GHz})$ bands, the MIMO antenna project can perform more efficiently with stability above $-17.5 \mathrm{~dB}$ for the low band and $-20 \mathrm{~dB}$ for the high band. The proposed dual-band four-antenna MIMO module has been developed and tested, with strong agreement found between simulation and measurement practice. The two compact antenna pairs on both sides of the module surface are perpendicular to the module surface. The phantom hand and display panel function are also examined in terms of the quality of the MIMO antenna system.

Ikram, M., Wang, Y., Sharawi, M. S., \& Abbosh, A. [15] explained that the novel multiple-input multiple-output (MIMO) an array of inverted-F (PIFA) supported printed microphones are given. The array is configured for mobile $5 \mathrm{G}$ applications for the $28 \mathrm{GHz}$ band implementation. It includes of a 4-element MIMO antenna system from which it is appropriate to include 8 PIFA antennas containing an attached antenna array in each element. To generate the collection in the shape of a tapered T-junction, a 1 to 8 power divider/combiner is used. From 27.5 to $28.5 \mathrm{GHz}$, the pair operates at $28 \mathrm{GHz}$ with a $10 \mathrm{~dB} 1 \mathrm{GHz}$ bandwidth. The RO-4350B confirms the suggested specification with its height of $0.76 \mathrm{~mm}$ and dielectric constant of 3.48 . The substrate's measurements are $130 * 68 * 0.76 \mathrm{~mm}^{3}$, which are comparable to normal mobile dimensions. The decision to create consistency is quick and simple in shape, low profile and sleek appropriate for cell devices.

Jandi, Y., et al., [16] described that a model of a Modular dual band Antenna patch for the next $5 \mathrm{G}$ smartphones. The compact design antenna referring to, including the ground plane, has a $19 * 19 * 0.787 \mathrm{~mm}^{3}$ compact structure. The theoretical antenna, two of the mobile $5 \mathrm{G}$ communications candidate frequencies, functions at $10.15 \mathrm{GHz}$ 
and $28 \mathrm{GHz}$, with a configuration comprising $\mathrm{A} 5.51 \mathrm{~dB}$ gain at $10.15 \mathrm{GHz}$ and $8.03 \mathrm{~dB}$ at $28 \mathrm{GHz}$ and a pattern of directional radiation. The study was reviewed in order of Rogers 5880 with a thickness of $0.787 \mathrm{~mm}$ was designed for the antenna. Antenna geometry and boundary conditions it analyses the figure of Loss of return, the plot of gain, the plot of radiation pattern, and now the plot of VSWR.

Shi, X., Zhang, M., Xu, S., Liu, D., Wen, H., \& Wang, J. [17] described that a configuration for The use of folded monopole structures is known to be a lightweight dual band 8-element MIMO antenna. The MIMO antenna consists of the following folded monopole form of energy, each of which is symmetrically assembled on the substratum's orthogonal frame corners and is side-by-side, because without substrate clearance. In the three competing 3.4-3.6 GHz and 4.55-4.75 GHz bands, the folded dominance arms are used to resonate as radiation materials. Each have been two antenna elements can be used on both bands to decrease the mutual coupling bound to a $3 \mathrm{~mm}$ short neutral thread. By altering the framework of the antenna and the neutral side, dual band decoupling can be carried out. The isolation and efficiency of the MIMO antenna is enhanced and the ergodic channel capacities measured in both bands are very different compared to the $20 \mathrm{~dB}$ SNR of $41.2 \mathrm{bps} / \mathrm{Hz}$ and $40.3 \mathrm{bps} / \mathrm{Hz}$ compared to, respectively of the $8 * 8$ MIMO system.

Liu, Y., et al., [18] explained that, a multiple-output (MIMO) 8-port multiple-input array that functions on the cornerstone of the characteristic mode theory (CMT) of long-term evolution (LTE) band $42(3400-3600 \mathrm{MHz})$ is defined. Therefore, as a multi-antenna model with various demographic modes is cumbersome for the CMT to execute, a better course of action is advocated to properly provide invaluable national input and expand the envelope fibromyalgia relationship. While two antennas excite some of the same modes, the proposed technique is still interested in achieving the ECC of both antennas. The CMT provides a formal interpretation of geometries with multi-significant modes and obviously did so. With the use of the regression of the antenna structure and neutral ground, dual band decoupling can be operated. The MIMO antenna isolation and efficiency are strengthened and the power of the ergodic channel is quantified at $41.2 \mathrm{bps} / \mathrm{Hz}$ and $40.3 \mathrm{bps} / \mathrm{Hz}$ with a $20 \mathrm{~dB}$ SNR at both bands, respectively distantly related to the $8 * 8$ MIMO program's comfortable scenario. By using this model, it is necessary to reverse consideration of the mode number. Modal weighting coefficient (MWC) phases of the same for an arbitrary structure are most often found to be in phase or out of phase for modes excited by two ports. As a result, relatively similar modes stimulated by separate ports could be separated into two forms for the configuration of a multi-port MIMO array: equi-phase modules and anti-phase methods in the proposed system, and the trade-off of equi-phase modules and anti-phase methods in the proposed approach. Adapting the experimental setup to asymmetric configurations is, by extension, often easy. In order to entice the effectiveness of the proposed MIMO antenna architecture with the desired ECC, an 8-port MIMO array for $5 \mathrm{G}$ mobile applications is designed and introduced.

De Almeida, et al., [19] explained that many objects must engage with each other and with human beings in the future, calling for a wide availability of prospective programs and equipment. However, this question comes with a robust operation. This paper presents and discusses existing reports proposed to meet the criteria for the internet of 
things (IoT) and Quick Progress Coordination (mMTCC). It depends on the aspects of their physical layer and addresses vulnerabilities that need to be met by $5 \mathrm{G}$ networks in the IoT scenario in order to achieve excellent connectivity. Indeed, a brief tutorial is described on prospective technologies for radio accessibility for $5 \mathrm{G}$ networks, focused on IoT use cases. The advanced $5 \mathrm{G}$ waveforms answered to multiplexing of orthogonal frequency division (OFDM); 2) universal filtered multi carrier (UFMC); 3 ) multicarrier filter bank (FBMC); and 4) multiplexing of large time division (GFDM). There will still be pressure on the features that each radio access technology could include to solve the significant issues of the IoT.

Dai, L., et al., [20] explained that the hither to unknown expectations are described. Finally wireless transmission networks, in the fifth generation $(5 \mathrm{G})$. In recent years, multiple access (NOMA) has been non-orthogonal consistently reported as one of the possible ideas for solving the problem. Unlike in the contemporary orthogonal multiple access system (OMA) family, serving a broader the number of users than the number of orthogonal resource slots with the protection of non-orthogonal resource allocation is the essential distinguishing feature of NOMA. The sophisticated cancellation of inter-user interference at the protocols will solve this level to enhance the complexity of the receiver. Have included thorough evaluation of NOMA's original birth, latest advancement, and future research directions in this paper. Specifically, the main theory of NOMA will first be established, with a comparison of NOMA and OMA, particularly in the framework of the theory of detail. Then, by breaking them into three subgroups, namely, power domain and code domain NOMA, the general NOMA models are discussed. In particular, Their design principles and key components will be presented, and a detailed description of these NOMA schemes will be covered in terms of their spectral usefulness, continuous improvement, receiver complexity, etc. Finally, along with particular prospects and new performance expectations to counteract these, a collection of problematic active projects that should be overcome for NOMA will be demonstrated difficulties.

Pei, Y., et al., [21] said that the human survival issue in a cognitive radio network, a secondary application spectrum-sharing, is explored in this review from a system perspective. Considering an effective single-output multiple-input cognitive radio channels in which a multi-antenna SU transmitter (SU-Tx) communicates sensitive data to a licensed SU receiver (SU-Rx) when an eavesdropper is present on the predominant user's (PU) registered band. A quasi convex important understanding of determining the transmit covariance matrix under joint transmit power and interference power constraints to preserve capacity identifies the channel's secrecy results. To derive the sensor node covariance matrix, two numerical approaches are mentioned. The first methodology reconsiders the original quasi convex issue into a single convex semi definite programming (SDP) issue by examining its fundamental convexity, while the second method reveals the number between the stable CRN and the normal CRN, the original signal is transformed into a group of conventional statement that reflects optimization problems, which makes it easier to understand that the attractive option for secure MISO is beam construction CR channel. In order to mitigate three suboptimal measures are also proposed for computational complexity, namely scaled secret beam forming (SSB), potential secret beam forming (PSB) and the proposed cognitive 
beam forming $(\mathrm{PCB})$. And then, the outcomes of computer simulation show that the usability of secrecy can outweigh the three suboptimal frameworks below than other circumstance.

\section{Proposed work}

The platform paper predicts the implementation of 5G mobile network architecture, an All-IP model for interoperability in wireless and mobile computing. A user terminal (which in the new architecture plays a critical role) and an outcome variable, autonomous radio access technologies contain the content. Any of the technologies for radio access is now used by each terminal as an IP window to the outside world of the internet. From the inside of the mobile terminal, each radio access technology (RAT) should have its own radio interface. For instance, unless there are 7 different types of transactions you want to have access to. To be productive in testing for this architecture, the mobile terminal needed to have three distinct access-specific interfaces and have all of them active at the same time. Radio access technologies are mentioned at the first two OSI levels are determined by more or less QoS social support for internet access, which further ranges with access technology. Then the network layer is over the OSI-1 and 2 layers, and this layer is IPP in today's world of communication, either IPV4 or IPV6, independently whether its of frequency bands. The IP's target is to ensure adequate control data for the direct routing of IP packets tailored to specific application connections, sessions somewhere else on the internet between client applications and servers.

Packet routing should be completed in compliance with the applicable user's procedures. Application connections are made across sockets between customer and servers on the internet. End points for encrypted communications flows are internet sockets. Each web the socket is a special and different integration of ready or local transport port, IP address of the project and powerful contact port and protocol for transport category is local IP address and application. Considering that, in aimed at raising the integrated web socket uniquely based on the customer and the server's application, it is useful to create End-to-end communication between the sender and receiver using the internet protocol. This shows that, in the light of heterogeneous network interconnectivity, vertical shutdowns must be configured and kept constant between the respective radio technologies, the local IP, and the destination IP address. When a computer user is on at least one end of such an internal network, fixing these key pillars will ensure end-to-end handover integrity. Routing to the destination should be exclusive and vice versa, just use the same pathway to ensure whether packets are accurately laid out and to discourage or prevent packet losses.

Compatible IP frameworks are offered for The certain technology for radio access that is affordable to engage directly with either the required radio access. The IP address and net mask of each terminal IP interface and the parameters associated with IP packet routing through to the network are specified. Changing Access technology (i.e., vertical handover) might mean because after ordinary inter-system handover, the local IP address will modify. Then change some more Socket parameters and replacing the socket, locking the socket and setting up a new one. This solution is based 
largely on today's internet engagement. Suggest a better level of good note of network access functionality abstraction points of the process this catastrophic mistake in the protocol stack comprises higher layers. This module manages the right transparency and control functions or direct packet routing in the new architecture. along some very relevant frequency bands, requiring good synchronization with the functional network architecture that corresponds network abstraction and packet routing with the user terminal and provides in the proposed architecture functions in accordance with predetermined policies. This control system is, at the same time, an important component from which sales team can be determined for each technology for transmission. It is the internet side, the new infrastructure, and therefore, the internet side, that is the achievable infrastructure for testing the essential features of communication networks and providing a comprehensive view of the accuracy which can be provided from the user's applications to the malfunctioning internet server. Set-up protocol for new levels which really form the proposed architecture within the present protocol stack.

The abstraction level of the network will be provided by the existence of the terminal present IP tunnels over IP interfaces via many technologies of together to the terminal (i.e., mobile user). The tunnels between the user terminal and the process control, which is referred to as the regulation router and executes routing depending on specific policies, would be discussed in detail. This will construct a convenient tunnel mix relative the client will only set a local IP address for the number of client-side radio access technologies that will be developed by exposing sockets between mobile client applications and internet-based servers for internet communication. Policies whose rules are distributed through the virtual network layer protocol will appear to route IP packets via tunnels or find the desired tunnel. In this way, the appropriate abstraction of the network is performed first from client applications at the mobile terminal. The PN junction procedure for policy routing to the policy router it is managed conservatively after the establishment of global cyber security network technologies and initiated by the virtual network protocol mobile terminal (VPN). The introduction and implementation of tunnel connections is a critical component at the virtual network level.

\subsection{Summary of use-cases in the network architecture proposed}

Wireless network heterogeneity allows a person terminal to undertake a collection of access technologies likes and dislikes. For user applications, this decision delivers excellent conditions. In novel situations, the processes of achieving convergence are highly correlated with the mechanism for downloading. In other words, by initiating a Network-level interface, i.e. the user terminal's The need for the user application to contact the authorized application server really does seem to end from network access to resources. In addition to the virtual network layer, many cooperative software modules involves different functionalities are logically divided into, when this proposed new architecture, the virtual network layer conducts, including several studies pertaining to correspondence, confidentiality and synchronization of viewer application sessions. There are certain similarities between the virtual network layer's client and server tasks. 
Within the global architecture, each software module has a feature to deliver $5 \mathrm{G}$ heterogeneous systems with interoperability. On both sides of the architecture, the functionality of software modules is complemented by authoritarian interfaces with other modules and functional communication between peer protocol modules.

\subsection{Virtual network layer design in the proposed architecture}

On opposite sections (client and server), four common cooperative modules are interconnected. Therefore, the virtual network layer can identify throughout four basic functionalities. The first key characteristic of the virtual network layer is the abstraction of the network. This quickly to the cooperative teamwork between some of the software modules RAT-CCSM and MCCSM, designed to mask the level of IP seen by each technology for radio access. The client-side RAT-CCSM platform, in addition to this basic function, uses API interfaces to obtain additional modules of programming from lower-level radio access technologies to provide access to reasonable software modules specific comments. This connection is a device by the data are obtained for the construction of the Connectivity between all the different access technologies and the signal quality where obtained from the satellite radio access network projects. This also included the network and radio conditions of each radio access technology in module frameworks involved in the business world. Between the client-side RATCCSM and the system router's MCCSM module, tunnels are built. A driving transport the RATCCSM module must incorporate the process between the mobile client and the router mostly with policy.

Specifically, for the MCCSM system. The tunnel is widely recognized by proven IP connectivity of the relevant radio access technology. The tunnel's source IP address is the IP address obtained by building timbers via the challenge of the tunnel destination IP address to the hardware platforms, which is also seen from the mobile side in the uplink direction is the MCCSM protocol router software module loopback address.

The RAT-CCSM software module routinely maintains the Radio parameter (received signal level) and IP synchronization definitions for the same network, the state of each technology for radio access, meanwhile. Information on communication system is transported to another related software module, the major focus of that was to manage transmission between IP tunnels designated by tunnels through IP (the ITHC software module). In this module, the second product is component of the routing module, where routing is based on a policy based on a routing policy the information balanced manner. Their interaction results in the specification within the routing table of the needed Interfaces in tunnels. The technology module is closely related to the SPME authentication system management module, since it travels between the mobile client and the platform router for proper access control. The second step is to determine routing policies for the defined service level offered by service growth. Coordinated work on the client and server side between the software components of MQPBR and CQPBR enhances this possibility. The corresponding cooperation between the two modules focuses on the use of an advanced control protocol for forecasting developed specifically for this purpose. Within the routing matrix/table, its duty is to provide tunnel interfaces with applies to systems of routes or routing rules. 
The adjustments the client module of MQPBR is initiated and maintained in agreement with an ITHC framework. Similarly, the client IP address obtained from its client side is obtained by the software module is introduced by the MQPBR software module in the authentication and authorization framework-SPME. McIP might mark the actual determination of the mobile client IP address, where the SPME software module creates the client's heterogeneous network IP address and then transitions it to the MQPBR client module. Upper protocol ranges, including transport, session, and functionality thresholds, are represented by each user's McIP address, which is viewed as the IP network address for them. The ability of this software system to execute supervised routing between the two software systems, based on the application established by the client, is a key feature in contrast to other routing software applications. This module's routing table is constantly evolving, taking the place of a three-dimensional routing matrix in which the multiplexing interface specifications for each activated user programme are specified.

The complicating component involves the question of perception task techniques or threat identification and policies. The RAT-CCSM Module triggers the care plan module to perform proper user authentication and approvals to build tunnels to use the computer aided for the same approval. This technique is implemented by comparing "any "free" IP address received from a radio access network to the server's generic Email account on the other hand". In this case, RATCCSM transparently delegates these packets to the radio access application's network interfaces. The chances to begin between RAT-CCSM and MCCSM to set up or denouncing an IP tunnel after processing the consequence of an authentication and authorization process. In the client end, the user terminal contains all the data found in the local storage and inside the security software framework, while the policy router maintains the mobile client information in the optional software component, referred to as the $\mathrm{CPH}$, that may be part of a certain policy router in this $\mathrm{CPH}$ archive, all documentation, authentication parameters, and policies for each client. The policies and user parameters obtained represent a mobile network that is already obtained and stored in the $\mathrm{CPH}$ module from other systems and the RAT-CCSM module, the MQPBR, CQPBR and the IHTC module will now be made available. The RAT-CCSM module is needed to build the pipeline, the MQPBR and CQPBR modules are warned of the McIP address delegated, while IHTC is reminded of other Wind energy conversion policies that should complement it in the process of tipping over decisions.

The third aspect is linked to quality assurance. Procedures or security mechanism and regulations that are applying to users. On the client hand, the RAT-CCSM project made the corresponding module for proper user delivery authentication and request to gain the same approval with usage of the relevant software, build a tunnel. This, the process can be carried out By reason of any "free" IP address accumulated from wireless applications against with a designated on the other arm, the server's IP address. RATCCSM in this scenario, forward these packages directly to the packages transparently data transmission technologies network interfaces. After the receiving the results of an authorization and authentication The RAT-CCSM and MCCSM authentication process starts the IP tunnel supply chain or derides the application. On the customer premises, the user terminal collects all the facts from the inside security software 
module in a local storage, while the network router stores the mobile client module descriptions in an external software kit of a module known as $\mathrm{CPH}$ that can be part of the same related to risk (but, it is not mandatory). Provided a mobile device is specified by policies and user parameters, obtained material the RAT-CCSM module, as well as the modules MQPBR and CQPBR, and IHTCC operands are then made available and stored in $\mathrm{CPH}$ from any other cycle of operation. It is designated for a tunnel configured by the RAT-CCSM module, the MQPBR and CQPBR modules are alerted of the McIP address calculated, while other policies incorporated in the CHP are communicated to IHTC that should in the handover decision process, strengthen it.

The fourth application is applied to the mechanisms of management to calculate the parameters that identifying the consumer standard of support and experience applications. Cooperation this mechanism is activated On the QoS/QoE module's client side and on the QoS/QoE module's server side. The logo on the side of the mobile (client) terminal of this module is to regularly review the terminal side financial information meaning parameters of applications for radio access. As a result, the analyzed criteria include a fair overview of the service level that can be achieved from the existing radio access technologies between the user and the regulation router. Each networking technology produces results at the same time. The ITHC handover decision system uses algorithms to determine the validity of these factors.

The fifth usability of the network architecture is oriented only to the human existence and its positioning or inside heterogeneous wireless communication channel. This part is carried out in conjunction with the delivery of customer support by considering the inherent role of systems, products, and networks in the form of service or ingested under pre-defined policies professional from client computers. His consumer module as an ITHC software module, the side is portrayed and it has close contact with other network layer virtual software modules. The software module simultaneously processes data and also the RAT-CCSM software module (Tunnels and Tunnels Realized) each access technology's signal reception level. It would still be, sometimes, intrinsically linked to the module QoS/QoE from which intelligence about the main parameters of any radio access hardware used by the product is obtained.

\subsection{MISO technique}

The proposed scheme is discussed in this chapter on the account of the choosing of the algorithm used that depend on satellite communication using the MISO algorithm. Simple Block diagram of MISO technique is shown in Figure 2. The condition number for the actualization of higher channel condition states of a channel suggests that the channel is, as previously said, the BER is incompetently conditioned and thus improves. Therefore, mediocre channel state numbers have to have a pre coder used with better reliability at the expense of increased storage cost savings. The scheme delivers a pre-coding algorithm that positively impacts the channel condition number. Therefore, when a worse condition number is recorded, it is beyond the selected threshold, the LRAP THP pre coder is stronger with better selected emissions and more im predictability. If the condition number is lower than the well-conditioned channel threshold, it modifications the pre-coder to a simple version. 


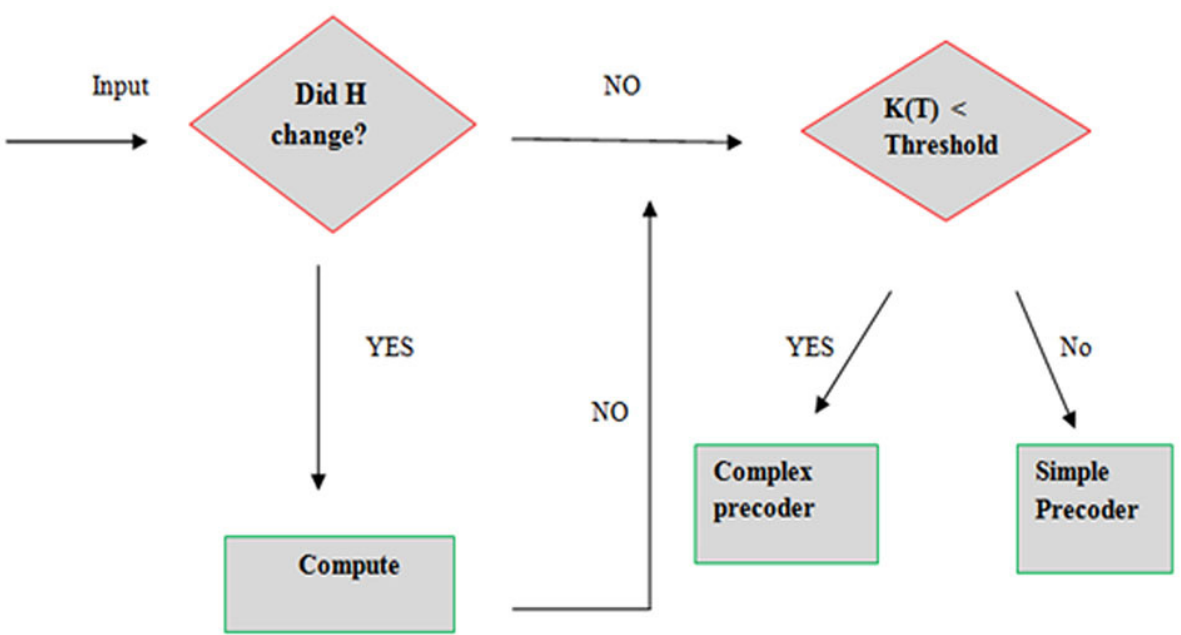

Fig. 2. Block diagram of MISO technique

The threshold was empirically selected in our simulations, taking into account the correlation coefficients of the mathematical circumstances. It is essential to use a low-complexity tactic to potentially estimate the number of scenarios. Depending on the use of the simpler ZF and THP methods. For the two combinations and the level of severity of the channel and percentage of application, the reliability was reported. The performance effects in terms of BER for separate instances. With the combination of ZF (40 percent) and LRAP THP, the worst channel success (deep shadow and elevation angle) of $10^{\circ}$ was measured for ZF, LRAP THP and the emergency vehicle (60 percent). In this comparison, the performance of the hybrid is roughly similar to the performance of the LRAP THP.

\section{$4 \quad$ Result}

The above algorithm is used to model a fabricated 4-antenna MISO array. Reduce the micro strip organizations to create that are already attached to the ground plane on the top of the partition. A microwave anechoic chamber calculates the S-parameters and far field radiation patterns of each antenna unit. The simulated and experimental loss of return are covered. Because with the symmetric structure of the 4-antenna MISO array, only those of Ant 1 and Ant 2 are given. According to fabrication mistakes, SMA welding complications and carbon emission, there are results in a change between the calculated and simulated consequences. The evaluated consequences, however, are still in good agreement with the simulated performance. Within the LTE band 466, the performance of ant 1 is substantially better compared to the simulated results. Other experimental representative S-parameters for the four-array assigned antenna-MISO. Only the probable loss of return of Ant I $(i=3,4)$ is received because of the structural symmetry. It reports that Ant 3 has 7.4 percent $(3380-3640 \mathrm{MHz})$ and 23.6 category 
(5140-6440 MHz) 10-dB impedance bandwidths, and 6.3 percent $(3400-3620 \mathrm{MHz})$ and 17.4 percent $(5120-6080 \mathrm{MHz})$ bandwidths for ant 4 . The experimental isolation (S12 and S23) is relatively larger compared with the simulations, Better than $11.2 \mathrm{~dB}$ though. The reported isolation on the narrow side between opposite antennas is compatible with the simulations, i.e. From inside $3500-\mathrm{MHz}$ and 5500-MHz channels $\mathrm{S} 15$, which is greater than $23 \mathrm{~dB}$, is typically shorter. At $3500 \mathrm{MHz}$ and $550000 \mathrm{MHz}$, the simulated and measured transformed far field radiation patterns could be seen in the $\mathrm{x}-\mathrm{y}$ plane, or. All others are compared to $50 \Omega$ matched loads, in additions to the antenna part under test. It can be established that the measured values agree very well for the simulated results. A relatively pure horizontal rotation can therefore be reached by the form of energy. In depth, at $3500 \mathrm{MHz}$, the Ant $\mathrm{I}=(1,2,3,4)$ actually radiates in the positive $\mathrm{x}$ direction, while the others' maximum radiation direction is specifically in the $\mathrm{x}$ direction. It is also necessary to detect related patterns of radiation at $5500 \mathrm{MHz}$. Therefore, a suitable omnidirectional property exhibits the proposed MISO antenna array, demonstrating the effectiveness of $5 \mathrm{G}$ mobile terminal technology. Here the below Figure 3 shows the capacity of antenna elements such as return loss and inter element isolation.

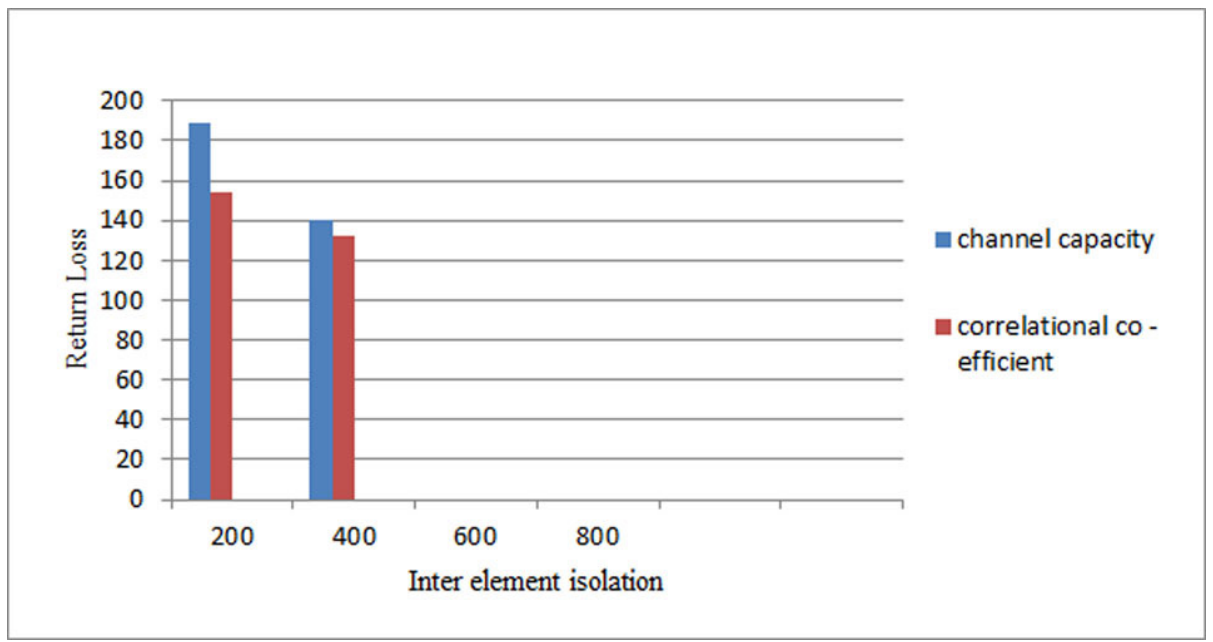

Fig. 3. Graphical representation of capacity of antenna elements

\section{Conclusion}

For theoretical 5G MISO applications based on slot SIRs, this paper to this a single-band four-antenna array. A return loss greater than $10 \mathrm{~dB}$, an inter-element impedance greater than $10.2 \mathrm{~dB}$, an ECC less than 0.2 , and a market value of the products greater than 61 percent were recorded for each antenna element band20, between the LTE band 52 and LTE band 52. The models and actual events are in good agreement. Between the two frequency bands, the channel bandwidth is expected to increase 
to $42.5 \mathrm{bps} / \mathrm{Hz}$ and $38.5-40.9 \mathrm{bps} / \mathrm{Hz}$. It is also easier to measure the head and human hands in the presence of the SAM for a low ECC and realistic MIMO reliability. The SAM head and human hands are in midst of, it is also feasible, to maintain a low ECC and sustainable MISO yield. Hence, a competitive candidate in mobile terminals for $5 \mathrm{G}$ multiband MISO applications could well be the proposed $4 * 4$ MISO antenna array. Which growth of communication systems networks are progressing towards higher data rates and an all-IP approach.

\section{References}

[1] De Looper, Christian (March 27, 2020). "What is 5G? The next-generation network explained". Digital Trends. Retrieved April 25, 2020.

[2] Forest interactive, Positive 5G Outlook Post COVID: What Does It Mean for Avid Gamers?, June 29, 2020. https://www.forest-interactive.com/newsroom/positive-5g-outlook-post-covid19-what-does-it-mean-for-avid-gamers/

[3] Horwitz, Jeremy (December 10, 2019). The definitive guide to 5G low, mid, and high band speeds. VentureBeat online magazine. Retrieved April 23, 2020. -19: What Does It Mean for Avid Gamers?. Forest Interactive. Retrieved November 13, 2020.

[4] Davies, Darrell (May 20, 2019). Small Cells - Big in 5G. Nokia. Retrieved August 29, 2020.

[5] Shatrughan Singh (March 16, 2018). Eight Reasons Why 5G Is Better Than 4G. Altran. Archived from the original on May 25, 2019. Retrieved May 25, 2019.

[6] Forum, C. L. X. (June 13, 2019). 1 Million IoT Devices per Square Km - Are We Ready for the 5G Transformation?. Medium. Archived from the original on July 12, 2019. Retrieved July 12, 2019.

[7] Segan, Sascha (December 14, 2018). What is 5G?. PC Magazine online. Ziff-Davis. Archived from the original on January 23, 2019. Retrieved January 23, 2019.

[8] Rappaport, T.S.; Sun, Shu; Mayzus, R.; Zhao, Hang; Azar, Y.; Wang, K.; Wong, G.N.; Schulz, J.K.; Samimi, M. (January 1, 2013). Millimeter Wave Mobile Communications for 5G Cellular: It Will Work!. IEEE Access. 1: 335-349. ISSN 2169-3536. https://doi. org/10.1109/ACCESS.2013.2260813

[9] Jump up to: a b Nordrum, Amy; Clark, Kristen (January 27, 2017). Everything you need to know about 5G. IEEE Spectrum magazine. Institute of Electrical and Electronic Engineers. Archived from the original on January 20, 2019. Retrieved January 23, 2019.

[10] K. L. Wong and J. Y. Lu. (Jan 2016). 8-antenna and 16-antenna arrays using the quad-antenna linear array as a building block for the 3.5-GHz LTE MIMO operation in the smartphone. Microwave Opt. Techn. Let., vol. 58, no. 1, pp. 174-181. https://doi.org/10.1002/mop.29527

[11] M. Y. Li, Y. L. Ban, Z. Q. Xu, J. H. Guo, and Z. F. Yu. (2018). Tri-polarized 12-antenna MIMO array for future 5G smartphone applications. IEEE Access, vol. 6, pp. 6160-6170. https://doi.org/10.1109/ACCESS.2017.2781705

[12] H. Xu et al. (2016). A compact and low-profile loop antenna with six resonant modes for LTE smartphone. IEEE Trans. Antennas Propag., vol. 64, no. 9, pp. 3743-3751. https://doi. org/10.1109/TAP.2016.2582919

[13] Abdullah, M., Kiani, S. H., \& Iqbal, A. (2019). Eight element multiple-input multiple-output (MIMO) antenna for 5G mobile applications. IEEE Access, 7, 134488-134495. https://doi. org/10.1109/ACCESS.2019.2941908

[14] Ren, Z., \& Zhao, A. (2019). Dual-band MIMO antenna with compact self-decoupled antenna pairs for 5G mobile applications. IEEE Access, 7, 82288-82296. https://doi.org/10.1109/ $\underline{\text { ACCESS.2019.2923666 }}$ 
[15] Ikram, M., Wang, Y., Sharawi, M. S., \& Abbosh, A. (2018, February). A novel connected PIFA array with MIMO configuration for 5G mobile applications. In 2018 Australian Microwave Symposium (AMS) (pp. 19-20). IEEE. https://doi.org/10.1109/AUSMS.2018.8346961

[16] Jandi, Y., Gharnati, F., \& Said, A. O. (2017, April). Design of a compact dual bands patch antenna for 5G applications. In 2017 International Conference on Wireless Technologies, Embedded and Intelligent Systems (WITS) (pp. 1-4). IEEE. https://doi.org/10.1109/ WITS.2017.7934628

[17] Shi, X., Zhang, M., Xu, S., Liu, D., Wen, H., \& Wang, J. (2017, March). Dual-band 8-element MIMO antenna with short neutral line for 5G mobile handset. In 2017 11th European Conference on Antennas and Propagation (EUCAP) (pp. 3140-3142). IEEE. https://doi. org/10.23919/EuCAP.2017.7928046

[18] Liu, Y., Ren, A., Liu, H., \& Wang, H. (2019). Eight-port MIMO array using characteristic mode theory for 5G smartphone applications. IEEE Access, 7, 45679-45692. https://doi. org/10.1109/ACCESS.2019.2909070

[19] de Almeida, I. B. F., Mendes, L. L., Rodrigues, J. J., \& da Cruz, M. A. (2019). 5 G waveforms for IoT applications. IEEE Communications Surveys \& Tutorials, 21(3), 2554-2567. https:// doi.org/10.1109/COMST.2019.2910817

[20] Dai, L., Wang, B., Ding, Z., Wang, Z., Chen, S., \& Hanzo, L. (2018). A survey of non-orthogonal multiple access for 5G. IEEE communications surveys \& tutorials, 20(3), 2294-2323. https://doi.org/10.1109/COMST.2018.2835558

[21] Pei, Y., Liang, Y. C., Zhang, L., Teh, K. C., \& Li, K. H. (2010). Secure communication over MISO cognitive radio channels. IEEE Transactions on Wireless Communications, 9(4), 1494-1502. https://doi.org/10.1109/TWC.2010.04.090746

[22] Politi-Georgousi, S., \& Drigas, A. (2020). Mobile Applications, An Emerging Powerful Tool for Dyslexia Screening and Intervention: A Systematic Literature Review. https://doi. org/10.3991/ijim.v14i18.15315

[23] Wongkhamdi, T., Cooharojananone, N., \& Khlaisang, J. (2020). E-Commerce Competence Assessment Mobile Application Development for SMEs in Thailand. https://doi. org/10.3991/ijim.v14i11.11358

[24] Rahmadya, B., Zaini, Z., \& Muharam, M. (2020). IoT: A Mobile Application and Multi-Hop Communication in Wireless Sensor Network for Water Monitoring. https://doi.org/10.3991/ ijim.v14i11.13681

\section{$7 \quad$ Authors}

Dr. T. Padmapriya is a Managing Director of Melange Academic Research Associates, Puducherry, India. She obtained her PhD in Pondicherry Engineering College, Puducherry, India. Her area of Research Interest is LTE, Wireless Networks. Email: padmapriyaa85@pec.edu

Dr. S. V. Manikanthan is a Director of Melange Academic Research Associates, Puducherry, India. His area of Research Interest is Wireless Sensor Networks. He has 21 years of experience in Anna University Affiliated Colleges and in Industrial Research Projects. Email: manikanthan.sv1@gmail.com

Article submitted 2021-05-08. Resubmitted 2021-06-15. Final acceptance 2021-06-15. Final version published as submitted by the authors. 